\title{
The Thermosphere of Titan
}

\author{
A. JAmes Friedson and Yuk L. Yung \\ Division of Geological and Planetary Sciences, California Institute of Technology
}

\begin{abstract}
The diurnal variation of the vertical structure of Titan's thermosphere is calculated through simultaneous solution of the equations of heat transfer and hydrostatic equilibrium. The temperature and density profiles are found above the mesopause. The dynamical response of the thermosphere to heating is for the most part neglected. Nevertheless, we are able to draw some interesting qualitative and quantitative conclusions regarding the vertical structure. Heating of the upper thermosphere occurs primarily through absorption of solar Lyman $\alpha$ radiation by methane, with an additional amount of heating $(\$ 20 \%)$ due to low-energy magnetospheric electron precipitation. The heat is conducted downward to the mesopause, where it is removed by IR cooling due principally to acetylene. The mesopause is found to occur where the density is $2.2 \times 10^{12} \mathrm{~cm}^{-3}(736 \mathrm{~km})$, and has a temperature of $\sim 110 \mathrm{~K}$. The exospheric temperature is unlikely to exceed $225 \mathrm{~K}$ in the course of a Titan day. The diurnally averaged exospheric temperature is in the range 187-197 $\mathrm{K}$ depending on the amount of magnetospheric electron heating that is included in the model. The amplitude of the diurnal variation is found to be $\$ 28 \mathrm{~K}$. We find that the vertical extent of the hydrogen cloud is too large to be explained in terms of simple thermal escape of hydrogen from a $\sim 225-\mathrm{K}$ exosphere, and we conclude that other processes must be important for populating or heating the neutral torus.
\end{abstract}

\section{INTRODUCTION}

As Voyager 1 passed through Titan's shadow, an occultation of the sun was observed at the morning and evening terminators by the ultraviolet spectrometer (UVS). Analysis of the occultation data has yielded the temperature and density near the exobase. The temperature at $3840 \mathrm{~km}$ (measured from the center of Titan) has been inferred to be $176 \pm 20 \mathrm{~K}$ at the evening terminator and $196 \pm 20 \mathrm{~K}$ at the morning terminator, while the $\mathrm{N}_{2}$ density at the same altitude is seen to be nearly the same at each terminator and equal to $2.7 \pm 0.2$ $\times 10^{8} \mathrm{~cm}^{-3}$ [Smith et al., 1982].

It is apparent from the UVS observation that the morning and evening exospheric temperatures do not differ by much and may in fact be equal. This suggests that there may be little diurnal variation of the exospheric temperature. It should be pointed out, however, that the exospheric temperature at the morning and evening terminators of Venus was also measured to be quite similar [Keating et al., 1980], yet the exospheric temperature there exhibits a strong dependence on local time. Consequently, it would be ill advised to conclude a priori that Titan's exospheric temperature does not substantially vary during one rotation period.

Our aim in the present study is to calculate the vertical structure of the thermosphere, down to the mesopause, as a function of local time. In so doing we have ignored any dynamical response of the thermosphere except that associated with the "breathing velocity" of constant-pressure surfaces. Hence the amplitude of any diurnal variation predicted by our model must be interpreted as an upper limit. In addition, the calculation of the vertical structure is appropriate only for Titan's equator.

Our task of modeling the temperature profile in the upper thermosphere is complicated by the fact that it is difficult to accurately quantify the heating due to precipitating magnetospheric electrons. Results of the Plasma Science experiment on Voyagers 1 and 2 [Bridge et al., 1981, 1982] have shown that conditions in the magnetospheric environment at

\section{Copyright 1984 by the American Geophysical Union.}

Paper number $3 A 1663$.

$0148-0227 / 84 / 003 \mathrm{~A}-1663 \$ 05.00$ the orbit of Titan are complex and highly variable. While the Voyager 1 UVS observation provided useful information on the energy flux of electrons incident on Titan at the time of the observation, the electron flux at other points along Titan's orbit is poorly constrained. Hence a model of the diurnal variation of the thermosphere which incorporates electron heating must make some initial, arbitrary assumption about the plasma conditions along the orbit.

The lack of any data concerning the rotation rate of the thermosphere becomes an additional source of uncertainty for the modeling. The Voyager 1 infrared interferometer spectrometer (IRIS) experimenters have inferred the presence of superrotating winds in the stratosphere, but there is no compelling reason to believe that this superrotation extends all the way up to the thermosphere. In view of the complexity of plasma conditions at Titan's orbit and of our lack of knowledge of the thermospheric rotation rate, we cannot hope to present a comprehensive quantitative model of the thermosphere. However, some aspects of the thermospheric structure, such as the location and temperature of the mesopause, are fairly insensitive to the uncertainties discussed above. In addition, we can set an upper limit to the exospheric temperature that can be achieved during a Titan day.

The thermal time scales that characterize the diurnal behavior of the thermosphere are discussed in section 2 . In section 3 we present the input model atmosphere used to calculate the temperature profile and discuss the dominant heating and cooling mechanisms in the thermosphere. The temperature profiles obtained by integrating the heat transfer equation with and without electron heating are presented and discussed in section 4. In section 5 we investigate what implications the derived exospheric temperatures have for the neutral hydrogen torus.

\section{Two Thermal Time Scales}

The way in which thermospheric temperatures vary in time can be understood in terms of two time scales, which are related to the two principal cooling mechanisms. The first time scale is the response time associated with nonlocal thermodynamic equilibrium (NLTE; vibrational relaxation) cooling due to acetylene and methane. It is roughly the time required for a given level in the thermosphere to relax to a new steady state 


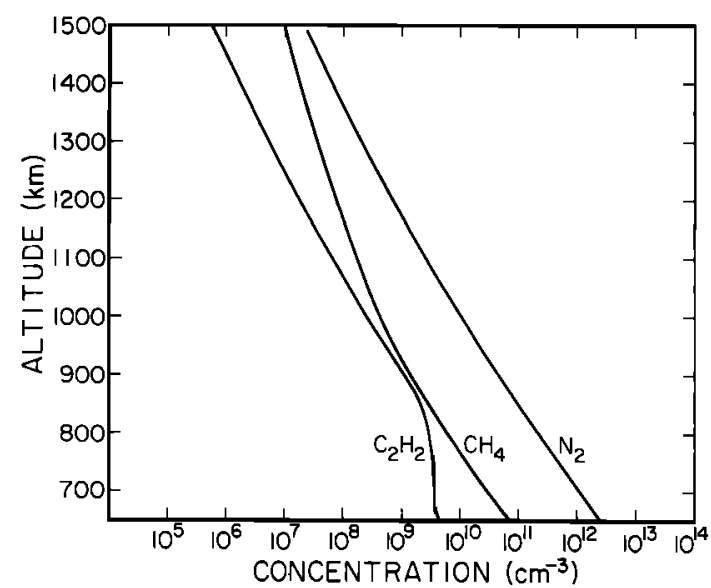

Fig. 1. Altitude profiles of $\mathrm{N}_{2}, \mathrm{CH}_{4}$, and $\mathrm{C}_{2} \mathrm{H}_{2}$ chosen as initial input for model calculations. These profiles are recalculated simultaneously with the temperature profile.

in response to a change in the heating, if the only cooling mechanism is NLTE cooling. This response time is given by

$$
\tau=\frac{\rho C_{P}}{\left.(\partial R / \partial T)\right|_{T}}
$$

where $\rho$ is the mass density at the level of interest, $C_{P}$ is the specific heat at constant pressure of $\mathrm{N}_{2}, R$ is the total NLTE cooling rate, and $T$ is the temperature. At all altitudes considered in our models, the response time $\tau \gtrsim 3$ years. Since the longest the thermospheric rotation period is likely to be is only 16 days, we may conclude that the temperature profile at any local time is not a steady state profile but contains a good deal of memory of the temperatures at past local times.

The second time scale of interest is simply the conduction relaxation time for the thermosphere above a given altitude level. It is given by

$$
\tau_{c} \sim L^{2} / \kappa
$$

where $L$ is a characteristic scale length of the system and $\kappa$ is the effective heat diffusivity. It is not immediately clear how to choose $L$ and $\kappa$ for an inhomogeneous medium, but it is natural to choose $L$ to be equal to the scale height at a given altitude and to choose $\kappa$ to be the heat diffusivity there. The time scale $\tau_{c}$ is then roughly the time required for conduction to bring the atmosphere above the altitude of inte est into equilibrium with the lower layers.

We may estimate the density of the level where the conduction relaxation time is comparable to the thermospheric rotation period. It is given by

$$
n \cong P k / m C_{P} H^{2}
$$

where $n$ is the number density, $P$ the thermospheric rotation period, $k$ the heat conductivity of $\mathrm{N}_{2}, m$ the mass of an $\mathrm{N}_{2}$ molecule, and $H$ the local scale height. If $P$ is taken to be 16 days (corresponding to rigid corotation), then $n \sim 8 \times 10^{10}$ $\mathrm{cm}^{-3}$, corresponding to an altitude of $\sim 860 \mathrm{~km}$ above the surface. Therefore we expect very little diurnal variation of the temperature below this altitude. The amplitude of the diurnal variation need not be small above this level. In our numerical calculations, we find that the actual level where the diurnal amplitude is $\sim 5 \mathrm{~K}$ lies at $n \simeq 5 \times 10^{10} \mathrm{~cm}^{-3}$, which tends to support the scaling arguments used here.

\section{Model}

For our model of the thermosphere we use the results of the UVS solar occultation experiment, as reported by Smith et al. [1982], together with the photochemical model produced by Yung et al. [1982]. Only three constituents of the upper atmosphere are important to the energy balance: $\mathrm{N}_{2}, \mathrm{CH}_{4}$, and $\mathrm{C}_{2} \mathrm{H}_{2}$. The mixing ratio of methane is taken to be $8 \%$ near the exobase and falls off to $\sim 3 \%$ in the lower thermosphere. The acetylene mixing ratio drops from a value of $2 \%$ near the exobase to only $0.2 \%$ in the lower thermosphere, in accordance with the best fit model presented by Smith et al. The initial input altitude profiles of these constituents are shown in Figure 1.

Two sources of energy are available to heat the thermosphere: solar energy and energetic magnetospheric electrons [Strobel and Shemansky, 1982]. The solar flux used in our model is taken from Mount and Rottman [1981]. The flux in Lyman $\alpha$ incident on Titan is $5.5 \times 10^{9}$ photons $\mathrm{cm}^{-2} \mathrm{~s}^{-1}$. The bulk of the solar heating occurs through absorption of Lyman $\alpha$ by methane. Acetylene also contributes to the heating through its absorption at wavelengths less than $2000 \AA$. Although nitrogen is an order of magnitude more abundant than methane, it only absorbs at wavelengths shortward of $1000 \AA$, where the solar flux is low. Hence it contributes only $\sim 10 \%$ to the solar heating.

Methane is dissociated at Lyman $\alpha$ via three channels [Slanger, 1982]:

$$
\begin{array}{lr}
\mathrm{CH}_{4}+h v \rightarrow{ }^{1} \mathrm{CH}_{2}+\mathrm{H}_{2} & 40 \% \\
\mathrm{CH}_{4}+h v \rightarrow \mathrm{CH}_{2}+2 \mathrm{H} & 50 \% \\
\mathrm{CH}_{4}+h v \rightarrow \mathrm{CH}+\mathrm{H}_{2}+\mathrm{H} & 8 \%
\end{array}
$$

The heating efficiency due to $\mathrm{CH}_{4}$ absorption is calculated for each channel separately, and the weighted average over the channels is then taken to yield a mean heating efficiency. In each channel, the kinetic energy of the dissociation fragments as well as $50 \%$ of the $\mathrm{CH}_{2}$ (or $\mathrm{CH}$ ) recombination energy contributes to the local heating. Once the ${ }^{1} \mathrm{CH}_{2}$ radical is formed, it is rapidly quenched in collisions with $\mathrm{N}_{2}$ to ${ }^{3} \mathrm{CH}_{2}$. Subsequently, two ${ }^{3} \mathrm{CH}_{2}$ radicals combine to produce $\mathrm{C}_{2} \mathrm{H}_{2}$ and $\mathrm{H}_{2}$ [Y $\mathrm{Y}$ ung et al., 1982]. Since $\mathrm{C}_{2} \mathrm{H}_{2}$ is chemically stable in the thermosphere, the reaction sequence terminates at this point. This set of reactions releases $2.8 \mathrm{eV}$ of heat, and we include $50 \%$ of this recombination energy, or $1.4 \mathrm{eV}$, with the kinetic energy of the dissociation fragments when the heating efficiency is calculated. The recombination energy of $\mathrm{H}$, however, does not contribute to the local heating, as $\mathbf{H}$ is slow to react compared with the transport time scale. The average heating efficiency over the three methane channels is found to be 0.35 at Lyman $\alpha$. It varies from a maximum of 0.47 at 1000 $\AA$ to 0.32 at $1300 \AA$. A similar analysis done for acetylene yields a mean heating efficiency of 0.45 between 1300 and 2000 $\AA$.

The solar heating is given by

$$
H(r, \theta)=\sum_{i} n_{i}(r) \int d \lambda \varepsilon_{i}(\lambda) \sigma_{i}(\lambda) F_{\infty}(\lambda) e^{-\tau(r, \lambda) x(\theta)}
$$

where $r$ is the radial distance from Titan's center; $n_{i}$ is the concentration, $\varepsilon_{i}$ the heating efficiency, and $\sigma_{i}$ the absorption cross section for constituent $i ; F_{\infty}(\lambda)$ is the incident solar flux per unit wavelength; $\tau$ is the total normal optical depth; and $\chi(\theta)$ is the Chapman function for solar zenith angle $\theta$. The 


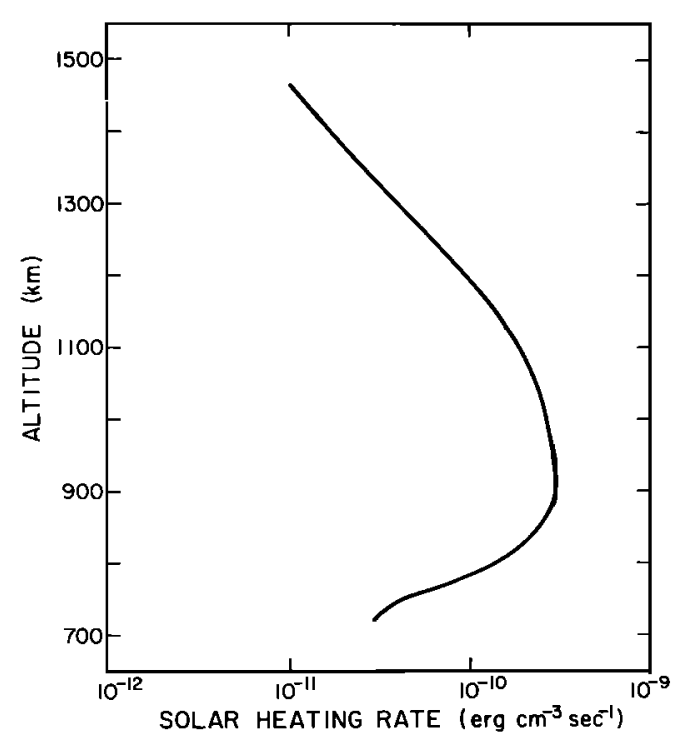

Fig. 2. Solar heating profile for solar zenith angle of $60^{\circ}$.

integration over wavelength is performed between $50 \AA$ and $2000 \AA$.

Another energy source for heating the thermosphere is derived from electron impact on $\mathbf{N}_{2}$. Some features of Titan's EUV emission spectra obtained during the Voyager 1 encounter suggest that some of the emission originates below the exobase [Strobel and Shemansky, 1982]. The emission is consistent with being caused by a secondary electron energy distribution that is roughly a Maxwellian with an electron temperature of $T_{e}=2 \times 10^{5} \mathrm{~K}$. This temperature corresponds to an average electron energy of $\sim 26 \mathrm{eV}$. Observation of the emission from the bright limb constrained the source altitude to be above $\sim 1100 \mathrm{~km}$. If photoelectrons produced $\sim 25 \mathrm{R}$ of $\mathrm{N}_{2}$ Lyman-Birge-Hopfield emission, then the hemisphericaveraged energy flux below the exobase was $\sim 0.01 \mathrm{erg} \mathrm{cm}^{-2}$ $\mathrm{s}^{-1}$ at the time of the observation [Strobel, 1983]. The altitude at which the source of this emission occurs, however, is still uncertain. Clearly, the actual electron energy flux incident on Titan should depend on the position of the satellite along its orbit. It is possible, in view of the complexity of the Saturnian magnetosphere at Titan's orbit, that this flux fluctuates substantially.

It is very difficult to calculate directly an effective heating efficiency for electron impact on $\mathbf{N}_{2}$ that would correctly take into account the numerous physical and chemical processes that determine the fraction of energy that is ultimately dissipated as heat. Generally, this quantity will lie somewhere between 0.3 and 0.7 . Since the electron energy flux below the exobase is probably uncertain by a factor of 2 or so, it seems pointless at this time to specify a unique value for the electron heating efficiency. We will lump the uncertainty in the electron energy flux and the heating efficiency together and treat their product, $\varepsilon \phi$, as an adjustable parameter. The value of $\varepsilon \phi$ is not well constrained by the data. Nevertheless, we have included a model run which incorporates a plausible amount of electron heating for illustrative purposes. We model the electron heating in the simple parametrized form

$$
E(r, \beta)=\varepsilon \phi(\beta) n(r) \sigma \exp [-\tau(r)]
$$

where

$$
\tau(r)=\int_{r}^{\infty} n\left(r^{\prime}\right) \sigma d r^{\prime}
$$

is the absorption path length for electron scattering, $\sigma$ is an "absorption" cross section, $\varepsilon$ is the electron heating efficiency, $\phi$ is the hemispherically averaged electron flux incident below the exobase, and $\beta$ is the angle between the Saturn-sun line and the position of Titan in its orbit. The cross section $\sigma$ is chosen to put the peak in the electron heating above the $1100-\mathrm{km}$ level. The value used for $\sigma$ is $4.2 \times 10^{-17} \mathrm{~cm}^{2}$. We consider the electron flux to have reached its peak during Voyager 1 closest approach $(\beta=0)$ and allow $\phi(\beta)=0$ on the nightside magnetosphere. This model is intended to roughly account for the dayside-nightside asymmetry in the plasma flux tube content observed by the Plasma Science experiments on Voyagers 1 and 2 [Bridge et al., 1981, 1982]. It is a crude approximation in that it ignores the longitudinal asymmetry in the electron deposition at Titan observed by the UVS experiment [Broadfoot et al., 1981; Strobel and Shemansky, 1982]. The value of $\varepsilon \phi(\beta=0)$ is taken to be $6 \times 10^{-3} \mathrm{erg}$ $\mathrm{cm}^{-2} \mathrm{~s}^{-1}$. Admitting a larger electron energy flux at the morning terminator causes the exospheric temperature to exceed the range allowed by the UVS observation.

The solar heating at a zenith angle of $60^{\circ}$ is plotted as a function of altitude in Figure 2. The peak electron heating as a function of altitude is shown in Figure 3.

Heat can be removed from the thermosphere by NLTE cooling. The cool-to-space approximation [Chamberlain and McElroy, 1966; Strobel and Smith, 1973] is quite adequate at all levels in our model. The $13.7-\mu \mathrm{m}$ band of acetylene and, to a smaller degree, the $7.7-\mu \mathrm{m}$ band of methane are responsible for the bulk of the NLTE cooling. Although methane is more abundant than acetylene in the thermosphere, acetylene dominates the NLTE cooling. This is true for two reasons: (1) the relaxation rate of the $13.7-\mu \mathrm{m}$ acetylene band in collisions with $\mathbf{N}_{2}$ is an order of magnitude greater than that of the methane 7.7- $\mu \mathrm{m}$ band, for the same temperature and pressure [Yardley et al., 1970; Hager et al., 1981], and (2) the lower energy associated with the $13.7-\mu \mathrm{m}$ transition gives it a higher probability of excitation relative to the $7.7-\mu \mathrm{m}$ transition by a factor of $\gtrsim 100$.

If we denote the total NLTE cooling rate per unit volume as $R(r, T)$, then

$$
R(r, T)=\sum_{i} \alpha_{i} h v_{i} \eta_{i}(T) e^{-h v_{i} / k T} f_{i}[n(r)]^{2} Y_{i}(r)
$$

Here $v_{i}$ is the frequency of a $13.7-\mu \mathrm{m}$ or $7.7-\mu \mathrm{m}$ transition, $T$ is the kinetic temperature of the ambient gas, $f_{i}$ is the mixing ratio of acetylene or methane, $n$ is the total density, $\eta_{i}$ is the

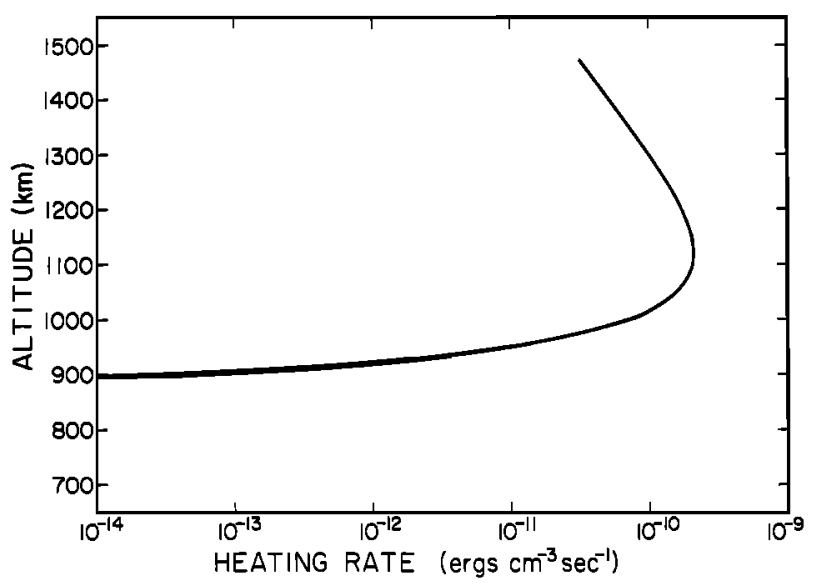

Fig. 3. Peak electron heating profile. 
collisional relaxation rate for the appropriate transition, $\alpha_{i}$ is the ratio of the statistical weights of the first excited state to the ground state, and $Y_{i}$ is the net collisional bracket for the transition. For a transition whose optical depth is $\lesssim 1 \%$ of its thermalization depth, $Y_{i} \simeq 1$ [Avrett and Hummer, 1965]. This is the case, at all altitudes of the model, for individual lines in the methane 7.7- $\mu \mathrm{m}$ band. Some lines in the acetylene $13.7-\mu \mathrm{m}$ band, however, will have optical depths of $\tau \sim 100$, or as much as $\sim 10 \%$ of the thermalization depth, at the lowest altitudes of the model. For these lines the value of $Y_{i}$ may differ substantially from unity. The value of $Y_{i}$ for the strongest lines is approximately 0.7 at the mesopause $(740 \mathrm{~km})$, and it decreases almost linearly to $\sim 0.1$ at the lower boundary of the model $(680 \mathrm{~km})$. We have determined, by numerical calculation, that the effect of setting $Y_{i}=1$ in equation (5) is to underestimate the mesopause temperature by $7 \mathrm{~K}$ and to overestimate the lapse rate in the vicinity of the mesopause. (In particular, the temperature gradient at the lower boundary of the model, as shown in Figure 5, is slightly superadiabatic. Allowing $Y_{i}$ to differ from unity in equation (5) causes the lapse rate to become subadiabatic.) For simplicity, we have set $Y_{i}=1$ in equation (5). Uncertainties associated with other aspects of the model are potentially more important.

The collisional relaxation rate of the $13.7-\mu \mathrm{m}$ band of $\mathrm{C}_{2} \mathrm{H}_{2}$ by $\mathrm{N}_{2}$ has been determined at room temperature [Hager et al., 1981]. We have not located any experimental data for this rate at other temperatures. Consequently, we have used data for the temperature dependence of $\mathrm{C}_{2} \mathrm{H}_{4}$ vibrational relaxation in $\mathrm{N}_{2}$ [Wang and Springer, 1977] and expect that these data should give an adequate description of the temperature dependence of the $\mathrm{C}_{2} \mathrm{H}_{2}-\mathrm{N}_{2}$ system. The collisional relaxation rate for $\mathrm{C}_{2} \mathrm{H}_{2}-\mathrm{N}_{2}$ is taken to be

$$
\eta_{\mathrm{C}_{2} \mathrm{H}_{2}}(T)=5.08 \times 10^{-14} T^{1 / 2} e^{-28 / T^{1 / 3}} \mathrm{~cm}^{3} / \mathrm{s}
$$

The collisional relaxation rate of the $\mathrm{CH}_{4} 7.7-\mu \mathrm{m}$ transition in $\mathrm{N}_{2}$ is given by [Wang and Springer, 1977]

$$
\eta_{\mathrm{CH}_{4}}(T)=1.02 \times 10^{-13} T^{1 / 2} e^{-56} / T^{1 / 3} \mathrm{~cm}^{3} / \mathrm{s}
$$

Heat is transported in the thermosphere by molecular conduction. For the conductivity of $\mathrm{N}_{2}$ we use

$$
k_{\mathrm{N}_{2}}(T)=42.7 T^{0.69}+2.61 T-355.6
$$

which fits the data to within $5 \%$ between $80 \mathrm{~K}$ and $500 \mathrm{~K}$ [Weast, 1967]. We neglect eddy heat conduction, since it is not clear to what extent the downward transport of heat due to eddies is able to balance or overcome the heat generated by viscous dissipation [Schoeberl et al., 1983; Walterscheid, 1981].

Given the heating and cooling rates, we calculate the vertical temperature profile as a function of local time by solving the time-dependent heat transfer equation

$$
\rho C_{P} \frac{\partial T}{\partial t}-\frac{1}{r^{2}} \frac{\partial}{\partial r}\left(r^{2} k_{\mathrm{N}_{2}}(T) \frac{\partial T}{\partial r}\right)=H(r, t)+E(r, t)+R(r, T)
$$

where $H(r, t)$ is the total solar heating rate, $E(r, t)$ is the electron heating rate, and $R(r, T)$ is the NLTE cooling rate. The specific heat at constant pressure, $C_{P}$, is used rather than $C_{V}$ in equation (9) in order to take account of the general expansion or contraction of constant-pressure surfaces in response to heating and cooling. This thermospheric "breathing" acts to reduce the diurnal temperature variation. Horizontal conduction and dynamical advection of heat are neglected in equation (9). These processes must reduce horizontal and vertical temperature gradients in the thermosphere, so the diurnal amplitude predicted by equation (9) must be considered as an upper limit.

Equation (9) must be supplemented with boundary conditions at the upper and lower boundaries of our model as well as with an initial temperature profile specified at a particular local time. The upper boundary condition is that the vertical temperature gradient must vanish above the exobase for all local times. We set the temperature to a constant value at the lower boundary, which occurs at an altitude of $680 \mathrm{~km}$. Choosing the temperature at $680 \mathrm{~km}$ to be independent of time is supported by the scaling arguments presented in section 2. Numerical solution of equation (9) has shown that the vertical structure obtained at and above the mesopause is insensitive to the particular value chosen for the temperature at the lower boundary. Specifically, the vertical temperature profile was found to be the same for lower boundary temperatures in the range $100-160 \mathrm{~K}$.

To guess an initial temperature profile for the morning terminator, we impose a steady state profile with a slightly reduced heating efficiency to account for the thermal inertia of the system. The validity of the initial guess is determined by the degree to which the solution returns to the initial profile after one rotation. Below $860 \mathrm{~km}$, where the diurnal temperature variation is expected to be negligible, the initial profile should be that associated with diurnally averaged heating conditions. A model run with diurnally averaged heating showed that the initial guess fulfilled this requirement.

The density profile at each local time is found simultaneously with the temperature profile by integrating the equation of hydrostatic equilibrium. The density at the lower boundary is chosen in order that the vertical profile at the morning terminator predicts a density at $1270 \mathrm{~km}$ of $2.6 \times 10^{8} \mathrm{~cm}^{-3}$, in agreement with the UVS observation. The mixing ratios of the minor species are held fixed during the diurnal run.

Equation (9) is integrated numerically by a finite difference relaxation technique. The vertical resolution is $8 \mathrm{~km}$; hence there are at least six steps per scale height in the model. The validity of the numerical solutions is checked by requiring energy to be conserved both locally and globally.

\section{Results AND Discussion}

The vertical structure of the thermosphere is found through simultaneous solution of the heat transfer equation and the equation of hydrostatic equilibrium. The value of the total density at the lower boundary is fixed by the condition that the density at $1270 \mathrm{~km}$ on the morning terminator agrees with the UVS solar occultation observation.

We have considered two model runs which differ only in the sources of heating for the thermosphere. Both models assume the rotation period of the thermosphere to be equal to Titan's orbital period (16 days). The first model considers the effects of solar heating alone, while the second introduces some amount of magnetospheric electron heating which might be representative of the conditions at Titan's orbit. The product $\varepsilon \phi(\beta=0)$ for the electron heating is taken to be $6 \times 10^{-3} \mathrm{erg}$ $\mathrm{cm}^{-2} \mathrm{~s}^{-1}$. For a hemispherically averaged electron energy flux below the exobase of $0.01 \mathrm{erg} \mathrm{cm}^{-2} \mathrm{~s}^{-1}$, this corresponds to an electron heating efficiency of 0.6 . It must be emphasized here that the actual amount of electron heating at Titan and its diurnal variation are highly uncertain. The true value of 


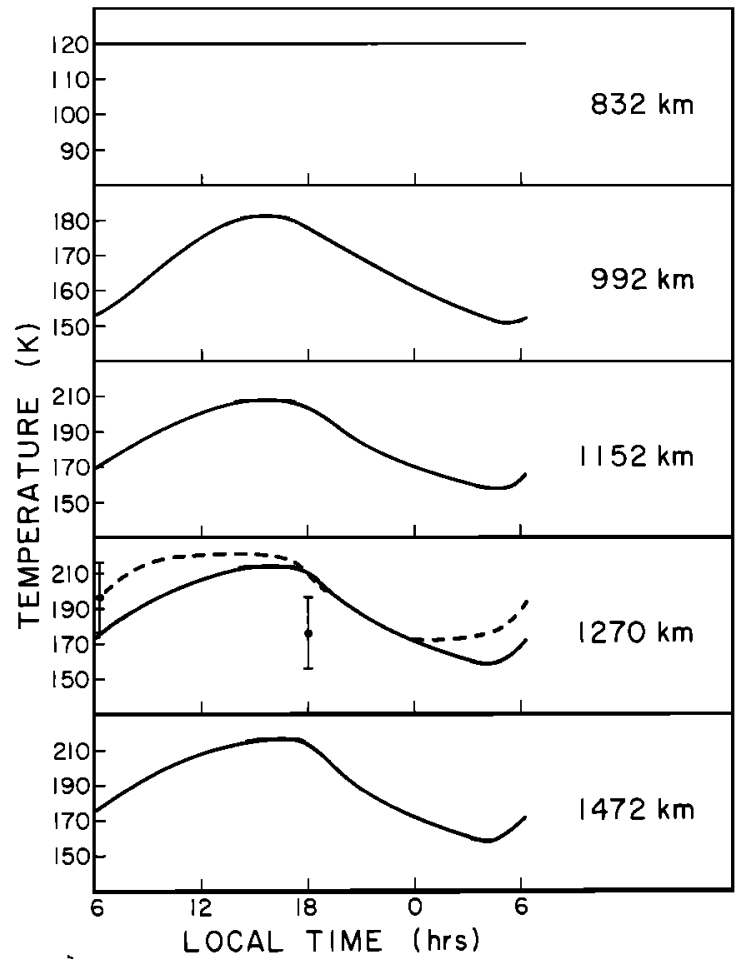

Fig. 4. Diurnal temperature variation at five altitudes, solar heating only. The dashed curve for $1270 \mathrm{~km}$ is for the model which includes electron heating.

$\varepsilon \phi(\beta=0)$ could be negligible, or it could be twice the value used in the model. Our purpose in including this model is to determine what, if any, effect the inclusion of an electron heat source has on the conclusions we make in this study.

Figure 4 shows the diurnal temperature variation at five altitudes when solar EUV radiation is the only source of heating. The data points shown on the curve for the $1270-\mathrm{km}$ level represent the UVS solar occultation measurements at each terminator. The peak exospheric temperature is $216 \mathrm{~K}$ and occurs at $\sim 1600$ hours local time, while the minimum of 159 $\mathrm{K}$ occurs at $\sim 0400$ hours. The diurnal amplitude of the exospheric temperature is $\Delta T \sim 30 \mathrm{~K}$. Also shown in Figure 4 is the diurnal variation at $1270 \mathrm{~km}$ when electron heating is included. The peak exospheric temperature is $\sim 6 \mathrm{~K}$ warmer for this case than for the solar heating case. Both models predict a temperature at $1270 \mathrm{~km}$ on the evening terminator which is $15 \mathrm{~K}$ warmer than the highest temperature allowed by the UVS observation. The dynamical response of the thermosphere or superrotation would be expected to reduce the diurnal amplitude of the temperature variation. Hence it is possible that winds or superrotation of the thermosphere could explain the discrepancy at the evening terminator.

The diurnal average temperature and density profiles for solar heating only are shown in Figure 5. These profiles were found as solutions to the diurnal average of equation (9) and to the equation of hydrostatic equilibrium. In order to fit the observed density at $1270 \mathrm{~km}$ on the morning terminator, we find that the density at the mesopause must be $n_{m}=2.3$ $\times 10^{12} \mathrm{~cm}^{-3}$. The mesopause is located at an altitude of 736 $\mathrm{km}$ and is quite cold, $T_{m}=104 \mathrm{~K}(110 \mathrm{~K}$ if we include opacity effects; see equation (5)). The diurnally averaged exospheric temperature is $187 \mathrm{~K}$. The diurnally averaged exospheric temperature is $\sim 10 \mathrm{~K}$ warmer than this when electron heating is included. The mesopause location and temperature are the same in both models and are therefore insensitive to the assumptions made concerning electron heating.

Superrotation of the thermosphere would tend to reduce the amplitude of the diurnal variation and tend to increase the time lag of the response to the heating. If there is a diurnal temperature variation in the thermosphere, the average cooling rate at each level is greater than the cooling rate in a thermosphere with the same average temperature but no diurnal variation. This arises from the nonlinear dependence of the cooling rate on temperature. As a consequence, a superrotating thermosphere with little diurnal temperature variation should exhibit a slightly higher diurnally averaged exospheric temperature than a slower rotator. In the limit where Titan's thermosphere is considered to rotate so fast as to not experience any diurnal variation, the average exospheric temperature is $\simeq 190 \mathrm{~K}$ when only solar heating is included. If these models come close to describing the actual heating conditions at Titan, it appears unlikely that the exospheric temperature exceeds $225 \mathrm{~K}$. A fluctuation in electron heating conditions (a local magnetospheric "storm," for example) could temporarily raise the exospheric temperature above this limit; however, the diurnal average of the exospheric temperature should stay below $225 \mathrm{~K}$.

\section{THE HydROgen TORUS}

The neutral hydrogen torus orbiting Saturn has been reported to extend radially between $8 R_{s}$ and $25 R_{s}$ and to have a vertical half thickness of 6-8 $R_{s}$ [Sandel et al., 1982]. It is interesting to consider to what extent the morphology of the torus is determined by simple Jeans escape of hydrogen from Titan.

In order to understand the torus geometry it is necessary to know first whether or not collisions between atoms are important to the dynamics of the cloud. Recent data of the flux of neutral particles, detected by the low-energy charged particle detector aboard Voyager 2 during the preencounter phase, have been used to set an upper limit of $100 \mathrm{~cm}^{-3}$ for the total neutral density in the torus (W. H. Ip, unpublished data, 1982). The mean free time for collisions, $\tau_{c}$, is of order $\tau_{c} \sim(n \sigma v)^{-1}$, where $n$ is the torus density, $\sigma$ is the collisional cross section, and $v$ is the average relative velocity between atoms. The rela-

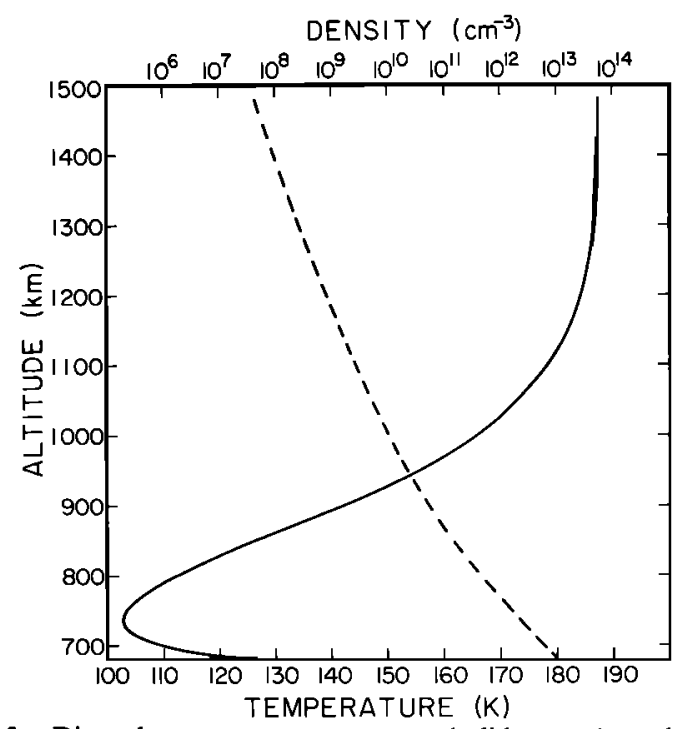

Fig. 5. Diurnal average temperature (solid curve) and density (dashed curve) profiles for solar heating. 
tive velocity between atoms is approximately $\sim e \Omega a$, where $\Omega$ is the orbital frequency, $e$ the eccentricity, and $a$ the semimajor axis of a typical particle orbit. Adopting values for $a$ and $\boldsymbol{\Omega}$ appropriate for particles near Titan, $\sigma \simeq 10^{-15} \mathrm{~cm}^{2}$, and $n \simeq 100 \mathrm{~cm}^{-3}$, the mean free collision time is of order $\tau_{c} \simeq$ $2 \times 10^{7} 1 / e \mathrm{~s}$. Thus for eccentricities of $\$ 0.2$, the collision time is greater than the torus lifetime of $10^{8} \mathrm{~s}$ estimated by Bridge et al. [1982]. A collisionless treatment for the torus would then be appropriate. Due to the uncertainties involved in this analysis, however, the question of the importance of collisions in the torus must be left open for the present.

Smyth [1981] used a detailed computer model to study the structure of a collisionless torus. For a mean emission velocity for $\mathrm{H}$ atoms of $2.0 \mathrm{~km} \mathrm{~s}^{-1}$, corresponding to an exospheric temperature of $\sim 240 \mathrm{~K}$, the vertical half thickness of the torus was found to be $1.5 R_{s}$. This is considerably less than the 6-8 $R_{s}$ inferred from the Voyager 1 and 2 UVS data. Therefore if the torus is collisionless, it is much thicker than can be explained by simple thermal escape from a $\sim 225-\mathrm{K}$ exosphere.

The same conclusion can be reached if the torus is collision dominated. A vertical scale height of $8 R_{s}$ implies a cloud temperature of $260 \mathrm{~K}$ [Sandel et al., 1982]. This is warmer than the 225-K upper limit we have set for the exospheric temperature. More complicated processes than thermal escape must be involved in forming a torus with half thickness $\sim 8$ $R_{s}$. Among the viable alternatives are nonthermal escape from Titan, gravitational scattering in the torus, local heating in the torus, or perhaps a source of neutral hydrogen other than Titan.

The diurnal variation of Titan's thermospheric temperature and density profiles has been calculated by simultaneously solving numerically the heat transfer equation and the equation of hydrostatic equilibrium. It is found that the exospheric temperature is unlikely to exceed $225 \mathrm{~K}$ during a Titan day. Its diurnally averaged value lies in the range $187-197 \mathrm{~K}$. The amplitude of the diurnal variation is $\$ 30 \mathrm{~K}$. The mesopause is fairly cold, $\sim 110 \mathrm{~K}$, and is located at a density of $2.3 \times 10^{12}$ $\mathrm{cm}^{-3}(736 \mathrm{~km})$. This density is greater than that expected at $736 \mathrm{~km}$ if Titan's upper atmosphere were isothermal above $190 \mathrm{~km}$ with a temperature of $170 \mathrm{~K}$. This suggests that the temperature reaches a local maximum, warmer than $170 \mathrm{~K}$, somewhere between $200 \mathrm{~km}$ and $700 \mathrm{~km}$ altitude. The vertical extent of the hydrogen torus is too large to be explained by simple thermal escape from a $\sim 225-\mathrm{K}$ exosphere. We conclude that other mechanisms must be more important in populating the torus.

Acknowledgments. The authors wish to thank A. P. Ingersoll, M. Allen, M. E. Summers, and G. R. Gladstone for helpful discussions. This research was supported by NASA grant NSG 7376 to the California Institute of Technology. Contribution 3907 from the Division of Geological and Planetary Sciences, California Institute of Technology.

The Editor thanks R. E. Hartle and D. Strobel for their assistance in evaluating this paper.

\section{REFERENCES}

Avrett, E. H., and D. G. Hummer, Non-coherent scattering, Mon. Not. R. Astron. Soc., 130, 295, 1965.
Bridge, H. S., J. W. Belcher, A. J. Lazarus, S. Olbert, J. D. Sullivan, F. Bagenal, P. R. Gazis, R. E. Hartle, K. W. Ogilvie, J. D. Scudder, E. C. Sittler, A. Eviatar, G. L. Siscoe, C. K. Goertz, and V. M. Vasyliunas. Plasma observations near Saturn: Initial results from Voyager 1, Science, 212, 217, 1981.

Bridge, H. S., J. W. Belcher, A. J. Lazarus, R. L. McNutt, J. D. Sullivan, P. R. Gazis, R. E. Hartle, K. W. Ogilvie, J. D. Scudder, E. C. Sittler, A. Eviatar, G. L. Siscoe, C. K. Goertz, and V. M. Vasyliunas, Plasma observations near Saturn: Initial results from Voyager 2, Science, 215, 563, 1982.

Broadfoot, A. L., B. R. Sandel, D. E. Shemansky, J. B. Holberg, G. R. Smith, D. F. Strobel, J. C. McConnell, S. Kumar, D. M. Hunten, S. K. Atreya, T. M. Donahue, H. W. Moos, J. L. Bertaux, J. E. Blamont, R. B. Pomphrey, and S. Linick, Extreme ultraviolet observations from Voyager 1 encounter with Saturn, Science, 212, 206, 1981.

Chamberlain, J. W., and M. B. McElroy, Martian atmosphere: The Mariner occultation experiment, Science, 152, 21, 1966.

Hager, J., W. Krieger, and J. Pfab, Collisional deactivation of laserexcited acetylene by $\mathrm{H}, \mathrm{HBr}, \mathrm{N}_{2}$, and $\mathrm{CO}, J$. Chem. Soc. Faraday Trans., 77, 469, 1981.

Keating, G. M., J. Y. Nicholson III, and L. R. Lake, Venus upper atmosphere structure, J. Geophys. Res., 85, 7941, 1980.

Mount, G. H., and G. J. Rottman, The solar spectral irradiance 1200 3184 angstrom near solar maximum: July $15,1980, J$. Geophys. Res., 86, 9193, 1981.

Sandel, B. R., D. E. Shemansky, A. L. Broadfobt, J. B. Holberg, G. R. Smith, J. C. McConnell, D. F. Strobel, S. K. Atreya, T. M. Donahue, H. W. Moos, D. M. Hunten, R. B. Pomphrey, and S. Linick, Extreme ultraviolet observations from the Voyager 2 encounter with Saturn, Science, 215, 548, 1982.

Schoeberl, M. R., D. F. Strobel, and J. P. Apruzese, A numerical model of gravity wave breaking and stress in the mesosphere, $J$. Geophys. Res., 88, 5249, 1983.

Slanger, T. G., Photodissociation channels at $1216 \AA$ for $\mathrm{H}_{2} \mathrm{O}, \mathrm{NH}_{3}$, and $\mathrm{CH}_{4}$, J. Chem. Phys., 77, 2432, 1982.

Smith, G. R., D. F. Strobel, A. L. Broadfoot, B. R. Sandel, D. E. Shemansky, and J. B. Holberg, Titan's upper atmosphere: Composition and temperature from the EUV solar occultation results, $J$. Geophys. Res., 87, 1351, 1982.

Smyth, W. H., Titan's hydrogen torus, Astrophys. J., 246, 344, 1981.

Strobel, D. F., Upper atmosphere and torus, in Saturn, edited by T. Gehrels, University of Arizona Press, Tucson, in press, 1983.

Strobel, D. F., and D. E. Shemansky, EUV emission from Titan's upper atmosphere: Voyager 1 encounter, J. Geophys. Res., 87, 1361, 1982.

Strobel, D. F., and G. R. Smith, On the temperature of the Jovian thermosphere, J. Atmos. Sci., 30, 718, 1973.

Walterscheid, R. L., Dynamical cooling induced by dissipating internal gravity waves, Geophys. Res. Lett., 8, 1235, 1981.

Wang, J.C.F., and G. S. Springer, Vibrational relaxation times in $\mathrm{CH}_{4}-\mathrm{N}_{2}$ and $\mathrm{C}_{2} \mathrm{H}_{4}-\mathrm{N}_{2}$ mixtures, Prog. Astronaut. Aeronaut., 51, $849,1977$.

Weast, R. C. (Ed.), Handbook of Chemistry and Physics, CRC, Cleveland, 1967.

Yardley, J. T., M. N. Fertig, and C. B. Moore, Vibrational deactivation in methane mixtures, J. Chem. Phys., 52, 1450, 1970.

Yung, Y. L., M. Allen, and J. P. Pinto, Photochemistry of methane, nitrogen and carbon monoxide on Titan: Abiotic synthesis of organic compounds, paper presented at the First Symposium on Chemical Evolution and the Origin and Evolution of Life, NASA Ames Research Center, Moffett Field, Calif., Aug. 2-4, 1982.

A. J. Friedson and Y. L. Yung, California Institute of Technology, Division of Geological and Planetary Sciences, Pasadena, CA 91125.

(Received August 30, 1982; revised October 10, 1983; accepted October 11, 1983.) 